\title{
ASO Author Reflections: The Association Between Low-Risk DCIS and Screening Ultrasound over the Past 10 Years
}

\author{
Si Eun Lee, MD, $\mathrm{PhD}^{\mathbf{1}}$, and Hee Jung Moon, $\mathrm{MD}, \mathrm{PhD}^{2}$ \\ ${ }^{1}$ Department of Radiology, Yongin Severance Hospital, Yonsei University College of Medicine, Yongin, Korea; \\ ${ }^{2}$ Department of Radiology, Wonju Severance Christian Hospital, Yonsei University Wonju College of Medicine, Wonju, \\ Korea
}

\section{PAST}

The proportion of ductal carcinoma in situ (DCIS) among breast cancers has increased from $3 \%$ to $25 \%$ on the strength of population-based mammography. ${ }^{1}$ On the other hand, as patients diagnosed with DCIS generally show excellent long-term survival, nowadays low-risk DCIS is under investigation for the possibility of active surveillance rather than surgical treatment in three ongoing trials. ${ }^{2}$ Considering these changes, it is meaningful to know the chronological trend of DCIS from clinical, pathological, and radiological perspectives.

\section{PRESENT}

Over the past 10 years, the detection rate of DCIS by ultrasound has markedly increased from 2.7 to $31.0 \%$. $^{3}$ During the same period, low-to-intermediate nuclear grade rates and the absence of comedonecrosis rates have also increased significantly, along with the rates of conservation surgery. ${ }^{3}$ This suggests a possible association between DCIS detected by ultrasound and low-risk DCIS, followed by an issue of overdiagnosis.

(C) Society of Surgical Oncology 2021

First Received: 17 June 2021

Accepted: 17 June 2021;

Published Online: 7 July 2021

H. J. Moon, MD, $\mathrm{PhD}$

e-mail: minerva44u@gmail.com

\section{FUTURE}

With increasing low-risk DCIS, candidates for active surveillance may increase in the future. Radiologists must be aware of the impact of screening ultrasound on the increasing proportion of DCIS. In addition, for accurate triaging of low-risk DCIS, which is not yet clearly defined, we need to standardize the criteria encompassing clinical, pathological, and radiological factors.

FUNDING Dr. Moon is supported by supported by the Basic Science Research Program through the National Research Foundation of Korea (NRF) by the Ministry of Education 2018R1D1A1B07049378). The funders had no role in the study design, data collection and analysis, decision to publish, or preparation of the manuscript.

\section{DISCLOSURE}

CONFLICT OF INTEREST Dr. Lee reports no conflicts of interest.

\section{REFERENCES}

1. Esserman L, Yau C. Rethinking the standard for ductal carcinoma in situ treatment. JAMA Oncol. 2015;1:881-3.

2. van Seijen M, Lips EH, Thompson AM, et al. Ductal carcinoma in situ: to treat or not to treat, that is the question. Br J Cancer. 2019. https://doi.org/10.1038/s41416-019-0478-6.

3. Lee SE, Kim HY, Yoon JH, et al. Chronological trends of breast ductal carcinoma in situ: Clinical, radiologic, and pathologic perspectives. Ann Surg Oncol. 2021. https://doi.org/10.1245/s104 34-021-10378-3.

Publisher's Note Springer Nature remains neutral with regard to jurisdictional claims in published maps and institutional affiliations. 